\title{
The Flight Navigation Planning Based on Potential Field Ant Colony Algorithm
}

\author{
Zhao Jin*, Bin Yan and Run Ye \\ University of Electronic Science and Technology of China, Chengdu, China
}

\begin{abstract}
Path planning in complex environment is the main foundation of flights automation navigation. Therefore, this paper aims to propose an algorithm combined ant colony optimal algorithm with potential field heuristic information. The main implement method of this algorithm is to use the information between the environment and goal to build the heuristic elements. What's more, the method defines this information as initial pheromone and converses elements to guide the ants to find the shortest path. Thanks to the heuristic elements in algorithm , the PFACO algorithm has a better searching tendency than traditional ACO algorithm, and overcome disadvantage in ants blindness. Through the simulation, the results show that PFACO has a better tendency of convergence. Compared with ACO, the PFACO algorithm can find a shorter path in same convergence time.
\end{abstract}

\section{Keywords - path planning; Ant Colony Optimal; Artificial Potential Field; UAV}

\section{INTRODUCTION}

With the application of UAV (Unmanned aerial vehicle)been widely used in different fields, the autonomous navigation has been a popular point to research. Hence, path planning is the fundament of UAV automation navigation. Therefore, the skills of finding a collision-free and effective path should be necessary for UAV mission completed. Because of the flexible capability in VTOL (Vertical Take-off and Landing), rotary-wing UAV has been paid more attention in navigation inspection mission. Therefore, the protagonist of this paper is to use rotary-wing UAV to complete the mission of path planning.

The principle of path planning is to find a collision-free and optimal path between start point and goal. In general, path planning method can be separated into global planning and partial planning. And global path planning is based on global environment, the partial planning is an assist tool accompany with global methods. To solve the planning problems, the method firstly needs the mission known digital environment as constrain condition. Then, according to the cost equation, the ability of UAV and the given map to simulate the mission trajectory. In the end, send the analog data to the PC to apply in real world. Therefore, an accuracy data map and effective path planning algorithm is needed necessarily.

As far as we know, there are plenty of path planning algorithms been come up by scientists. Such as Dijkstra algorithm ${ }^{[1]}, A^{*}$ algorithm [2], Artificial potential field [3], genetic algorithm ${ }^{[4,5]}$, neural network algorithm ${ }^{[6]}$. And each algorithm shows the effective in path planning. As to the ACO has the advantage of getting feedback based on the UAV situation quickly and the ability of robustness, it been widely used in the path planning research. But the method also gained the disadvantages in searching speed and premature in path planning. The APF (Artificial Potential field) is a speedy obstacle avoidance algorithm, and can ensure the safety of UAV based on the gravitation and repulsion. But once the force of gravitation equals with the repulsion, the algorithm will suck into local minimum and stop forward. This paper main uses the idea of combination about ACO and APF. Firstly, it uses the ACO algorithm as main improve method to rebuild. Then, taking the potential field as heuristic factor in ACO transition probability to improve the ability in global searching. What's more, we also use the potential field to build initial pheromone field instead of random field to increase the speed of the method's rate of convergence. The result will be shown in the last to prove the effective of the PFACO algorithm.

The structure of this paper is shown as follows. In section II, the research on recently path planning methods and the improved ACO. Section III and section IV are the principles of ACO and APF. Section V and VI show the details about the idea of PFACO. From section VII, the results will prove the effectiveness of the algorithm. Last but not least, section VIII draws the conclusions.

\section{RELATED WORK}

Ant Colony Optimal algorithm is a multifactor algorithm created by M. Dorigo ${ }^{[7,8]}$. The ACO is a bionic algorithm based on ants' food searching and mainly used in TSP (Travelling Salesman problem). Therefore, it is equipped with strong robustness and positive feedback. What's more, ACO algorithm is a heuristic-driven method heavily depends on the density of pheromone and the importance of heuristic. So, the improvement in heuristic is an effective way to reinforce the ability in path searching.

Until now, thanks to the feature of compatibility, there are many combined ACO methods to improve the ability of path optimal searching. Adaptive ACO through ordinary ants, abnormal ants and random ants to search the relative path between start and goal notes to reduce the calculation of the whole methods ${ }^{[9,10]}$. The Chaos ACO is to add the chaos disturbance factor into ACO to overcome the local optimal and improve the path searching effectively ${ }^{[11]}$.

ACO-GA(Genetic-Algorithm) is a global searching algorithm to use the same GA phase crossover to find the local optimal solution phase to rise the value of smart path. 
Then, improve the global searching solution ${ }^{[12,13]}$. ACOPSO (Particle Swarm Optimization) is a method uses PSO to train the magnitude of ACO's parameters. By iteration, the PSO can find the best combination of parameters and rise the ability of ACO ${ }^{[14]}$. ACO-APF is a combination method, firstly through $\mathrm{PF}$ to weaken the fetters among the environment. Then, ACO is the main method to apply the mission of path searching, APF is to smooth the path and rise the ability of artificial ants ${ }^{[15]}$. Heuristics based ACO is a method to use semi-greedy heuristic and clustering phase transition rule to prove the searching ability ${ }^{[16]}$. What's more, Parallel ACO uses parallelized ants ACO and swarm intelligence technique to let method fit for any complex task [17].

\section{Ant Colony Optimal Method}

The ACO is an algorithm born from the idea of ants seek for foods. During the seeking path, the ants will deposit the pheromone to guide other fellows. With the time passing by, the pheromone will evaporate and become less. Therefore, the shorter path will be laid with heavier pheromone, and will get more possibility to be chosen. In the end, the path will restrain by the concentration of pheromone and get a shortest path. Therefore, the key points of ACO are the design of transition probability and the update of pheromone.

\section{A. Transition Probability}

During time $t$, the movement of $k_{\text {th }}$ ant transfers from point $i$ to point $j$ is related to the pheromone $\tau_{i j}(t)$ and the magnitude of heuristic $\eta_{i j}(t)$. The formula of transition probability will use equation (1) to choose which path to go when the $k_{\text {th }}$ ant reaches the maximum $\left[\tau_{i j}(t)\right]^{\alpha}\left[\eta_{i j}(t)\right]^{\beta}$ in $q_{0}$. Otherwise, the method will take roulette probability equation (2) as transition probability equation to choose next reach point.

$$
\begin{aligned}
& j= \begin{cases}\arg \max \left\{\left[\tau_{i j}\right]_{j \in \text { allowed }_{k}}^{\alpha}\left[\eta_{i]^{\beta}}\right\}\right. & \text { if } q \leq q_{0} \\
S & \text { otherwise }\end{cases}
\end{aligned}
$$

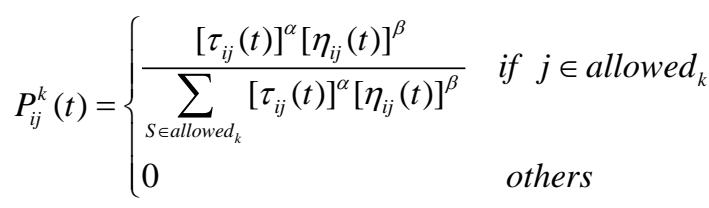

And the magnitude of heuristic can be expressed as:

$$
\eta_{i j}(t)=1 / d_{i j} \text {. }
$$

Where $\alpha$ represents for the importance of $\tau_{i j}$. The bigger $\alpha$ is, the more ants will choose the path with heavier pheromone. What's more, $\beta$ stands for the influence of heuristic, and the bigger $\beta$ will help ants to choose the closer point. In the end, $d_{i j}$ is the Euclidean distance between $i$ and $j$.

\section{B. The Update of Pheromone}

Like ants in real world, ants will release some pheromone to remark when passing away a path. And with time passing by, the concentration of pheromone will be weaker. Therefore, the strategy of the update of pheromone will be necessary to update the path ants passed to ensure the global of ACO. And the formula of the update of pheromone can be expressed as:

$$
\begin{gathered}
\tau_{i j}(t+1)=(1-\rho) \tau_{i j}(t)+\rho \Delta \tau_{i j}(t, t+1) \\
\Delta \tau_{i j}(t, t+1)= \begin{cases}Q / L_{k} & k_{t h} \text { ant pass though }(i, j) \\
0 & \text { otherwise }\end{cases}
\end{gathered}
$$

Where $\rho$ means the speed of the pheromone evaporation, and $\rho \in\left[\begin{array}{ll}0 & 1\end{array}\right]$ to avoid the unlimited iteration in updating the path. What's more, $\Delta \tau_{i j}(t, t+1)$ is the pheromone that ants left at $(i, j)$ in time $(t, t+1)$. Last, parameter $\mathrm{Q}$ represents for the intensity of pheromone.

\section{Potential Field Method}

Artificial potential field method is to build a potential field based on the mission environment, and the UAV can be influenced by gravitation and repulsion force among the environment to find a collision-free path. Where the gravitation is born from the working of goal point, the closer distance between UAV and destination, the bigger gravitation is. The force of repulsion is produced by obstacles, and the principle is same as the force of gravitation.

As mentioned earlier, the potential field is made by the force of gravitation $U_{\text {att }}$ and repulsion $U_{\text {rep. }}$. And $U_{\text {att }}$ is based on the location of the goal, $\mathrm{q}$ is the coordination in the potential. Therefore, the field of gravitation can be expressed as:

$$
U_{a t t}(q)=\frac{1}{2} \xi \rho_{g}^{2}(q)
$$

And $\rho_{g}=\left|q-q_{g}\right|$ is the distance between UAV and the goal, $q_{g}$ is the coordination of goal and $\xi$ is a parameter about gravitation.

As usual, the equation of the field of repulsion is employed as FIRAS function mentioned by Khabit. And it can be shown as:

$$
U_{\text {rep }}= \begin{cases}\frac{1}{2} \eta\left(\frac{1}{\rho(q)}-\frac{1}{\rho_{0}}\right)^{2} & \rho(q) \leq \rho_{0} \\ 0 & \rho(q) \geq \rho_{0}\end{cases}
$$

Where, $\eta$ is the constant about the force of repulsion. And $\rho_{0}$ is the range the obstacle can influence. Beyond the range of $\rho_{0}$, the UAV won't be influenced by the threats. Also $\rho(q)$ is the distance between the UAV and the threats. Combinate $U_{\text {att }}$ and $U_{\text {rep }}$, the UAV will away from the obstacles and move toward the goal. 


\section{V.Potential Field Ant Colony Optimal Method}

A. The Potential Field Heuristic

Because of the traditional ACO initial pheromones are same in everywhere, it always been the information of heuristic which makes the main influence in path finding. This way is similar with the GA (Greedy Algorithm), and may easier to be caught in local optimal. What's more, thanks to the positive feedback, the algorithm will follow the path with former information whether is wrong or not.

To overcome this problem, this paper uses the potential field among UAV and the point it reaches next to improve the density of heuristic. And then, bring the new $\eta_{i j}(t)$ to the transition probability equation (2) to find the next point. In order to avoid the local optimal in ACO, the PFACO method takes the advantage of decay parameter. The magnitude will get less with the iteration goes on. The new heuristic can be expressed as:

$$
\begin{gathered}
\theta=\angle\left(F_{\text {att }}+F_{\text {rep }}\right) \\
q=K \cdot \exp (\cos \theta) \\
\eta_{i j}(t)= \begin{cases}{\left[1-\frac{d_{i j}+L_{j g}}{\sum_{s \in \text { allowed }} d_{k}+L_{j g}}\right] \cdot q} & s \in \text { allowed }_{k} \\
0 & \text { otherwise }\end{cases}
\end{gathered}
$$

The method through calculating the angle among the location, goal and obstacles to build guidance cues. And it defines the angle $\theta$ to avoidance. What's more, the weight of potential field is $q$. In heuristic information, $d_{i j}$ is the Euclidean distance between point $i$ and point $j$. And $\mathrm{L}_{j g}$ is the potential field between next point with the goal. $N_{\text {camx }}$ is the maximum iteration, and $N_{c}$ is the iteration in this time. $\xi$ belongs to the decay parameter and $\xi>1$.

Thanks to the high adoptive and easy model of ACO and APF, it become easier to bring the idea of APF into ACO, and overcome the problem of local optimal in traditional ACO. What's more, improve the effective in ACO path searching.

\section{b. The Allocaton in Initial Pheronone}

In the earlier path planning, there is no difference of density in each note. So, the feedback of ACO works hard and the path searching with more blindness, also accompany with low effective. To solve this problem, the method concentrated on the allocation of initial pheromone. And the improved method defines that the notes between start and goal point are much bigger than other notes, it can be shown as:

$$
\tau_{i}= \begin{cases}C & i \in A \\ 1 & \text { ohterwise }\end{cases}
$$

In equation (8), $\tau_{i} \in \tau, \tau$ is the density of pheromone. $\mathrm{C}$ is a constant and bigger than $1 . \mathrm{A}=\left\{\min \left(g_{\text {start }}, g_{\text {end }}\right)\right.$, $\min \left(g_{\text {start }}\right.$, $\left.\left.g_{\text {end }}\right)+1, \ldots, \max \left(g_{\text {start }}, g_{\text {end }}\right)\right\}, g_{\text {start }}$ is the start note, $g_{\text {end }}$ is the goal note. Based on the Zero Theorem, the best path always exists among start and goal point. Therefore, it can be taken advantage to improve the searching purpose and effective of the algorithm.

\section{Improved Renew Pheromone}

When kth finished the moving from note $i$ to note $j$, the method would take the steps of local pheromone update. The update of equation as:

$$
\tau_{i j}(t+1)=(1-\lambda) \tau_{i j}(t)+\lambda \tau_{0}
$$

Where $\lambda$ is the local pheromone update parameter, and $\lambda \in(0,1) . \tau_{0}$ is a constant nearly to 0 . When $\tau_{i j}(t)<\tau_{\min }$, let $\tau_{i j}(t)=\left(\tau_{\min }+\tau_{\max }\right) \bullet 0.4$. Besides, if $\tau_{i j}(t)>\tau_{\max }$, let $\tau_{i j}(t)=\tau_{i j} \bullet \lambda . \tau_{\max }$ and $\tau_{\min }$ are the boundary the pheromone can be set to overcome the prematurity of the method, $\lambda$ is an constant , and been set as 0.6 .

When the PFACO finish an iteration, the algorithm will evaluation the global pheromone. In traditional ACO, the global update based on equation (3) to update. And Path ants went through will have a big influence for the decision in next iteration. It also means that the worse decision can make a much worse conclusion.

In order to improve the fault of the ACO, the paper uses the principle of MMAS (Min-Max Ant System) to find the best path and the worst path. In global pheromone updating, by weaken the worst path to let the ants in next iteration concentrate on finding path in best field found in former iteration. Through this solution, the worst will be receded gradually, and the better and be even better. Therefore, the improved pheromone update equation can be express as:

$$
\tau_{i j}(t+1)=(1-\rho) \tau_{i j}(t)-\varepsilon \frac{L_{\text {worst }}}{L_{\text {best }}}
$$

Where $\varepsilon$ is a constant, $L_{\text {worst }}$ is the longest path ant found in former iteration, and $L_{b e s t}$ is the most optimal path ant found in former iteration. The principle of MMAS can speed up the convergence of method effectivity. But the disadvantage of ACO may easier to let the algorithm into local optimal. By controlling the evaporation parameter $\rho$, ACO can improve the ability of global search. On the contrary, it will also cause the divergent of ACO. PFACO through the idea of self-adaption to adjust $\rho$, by adopting the information the former iteration left, the method can set suitable $\rho$ on time, and make best decision.

By setting up a threshold $R$, When the number of iteration smaller than $N_{c}, \rho$ will become less with the iteration continue. Once $N_{c}=\mathrm{R}$, the density of pheromone will be defined as $\rho_{0}$. It can be sown as:

$$
\rho(t+1)= \begin{cases}\max \left(\gamma \bullet \rho(t), \rho_{\min }\right) & N_{c} \neq R \\ \rho_{0} & N_{c}=R\end{cases}
$$


Where $\gamma$ is a constant lower than $1, \rho_{0}$ is the initial pheromone and equal as $1, \mathrm{R}$ is the threshold of iteration. What's more, $N_{c}$ is the iteration in time.

\section{The PRocess of Pfaco}

Based on the former formula, the process of PFACO can be showed in figure 1.

Step1: Setting up the initial parameters, such as: $\alpha, \beta$, $\tau_{\min }, \tau_{\max }$. And choose the start point $S$, goal point $g$. On the other hand, define the number of ants $M=50, N_{c, \max }=100$.

Step2: Allocating the initial pheromone. According to the formula(8), setting up the initial iteration pheromone randomly between start point $S$ and goal point $g$.

Step3: Start the PFACO path searching. Putting the ants into the start point. Then, calculating the $\theta, q, \mathrm{~L}_{\mathrm{ij}}, \mathrm{d}_{\mathrm{ij}}$ based on the formula (7), (8) and (9) to build PF heuristic information. Hence, let the PF heuristic information $\eta_{i j}$ into the transition probability (2). Finally, find the next accessible note $\mathrm{j}$ based on the equation (1) and (2) until all the ants reach destination. What's more, if the note $\mathrm{j}$ is accessible, put it into the tabu list in case of repeat choosing.

Step4: Local pheromone updating. When an ant finishes its searching, update the local pheromone based on equation(9) to improve the ability of global searching.

Step5: Global pheromone updating. When all the ants finish the path searching in iteration. Then, find the best and worst paths ants found. If the iteration $N=N_{c, \max }$, the method is over and $\mathrm{L}_{\text {best }}$ is the most optimal path PFACO found. If not, set up the parameter of global updating based on formula(11) and evaluate the former best path as equation(10).

Step6: Condition of method over. If $N=N_{c, \max }$, the method is over and output the best solution. Otherwise, clear the tabu list and continue the step3 until the iteration reaches the $N_{c \max }$.

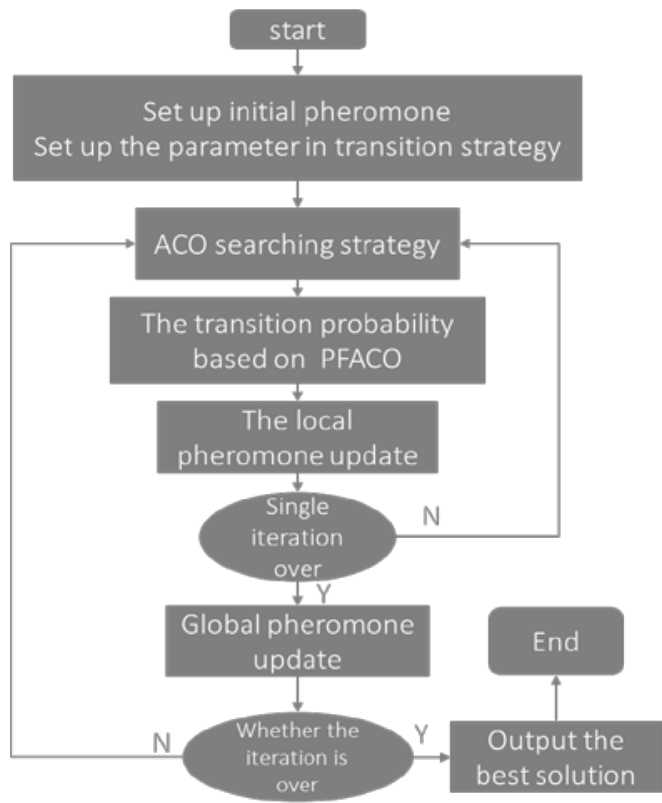

\section{FIGURE I. THE PROCESS OF PFACO}

\section{SIMULATION RESULTS}

For the purpose of proving the validity of PFACO, the paper uses a simulation to simulate the environment of UAV mission and show the process of UAV navigation. The simulation based on MATLAB R2016a and CORE i7 to set up Test Environment. And The Test Environment main content some rectangle obstacles as UAV navigation threats. Both ACO and PFACO set up parameters as $\alpha=0.5, \beta=0.5, \tau_{\min }=0.01, \tau_{\max }=15$, $\mathrm{K}=100, \mathrm{~N}_{\mathrm{c}, \max }=50$. The location of start point is $(1,-4,1)$ and the goal is $(5,17,3)$.

From figure 2, it shows that traditional ACO can totally satisfy the quest of collision-free. But the path still has space to optimize. What's more, the speed of convergence takes too long. The traditional ACO takes about $20_{\text {th }}$ iterations to reach convergence, and the graph shows that the method lacks the ability than global optimize.
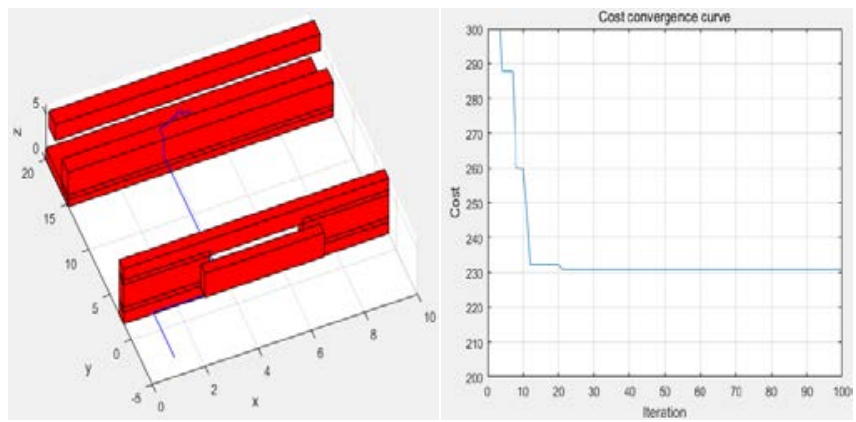

FIGURE II. ACO PATH PLANNING AND ITS CONVERGENCE GRAPH

Compared with ACO, the PFACO has a shorter path than traditional ACO based on figure4. Beides, the PFACO graph is more steepness, which means the method has a better convergence in path searching. And the PFACO can find a better path in 12 th iteration, speed up $40 \%$ with lower cost.
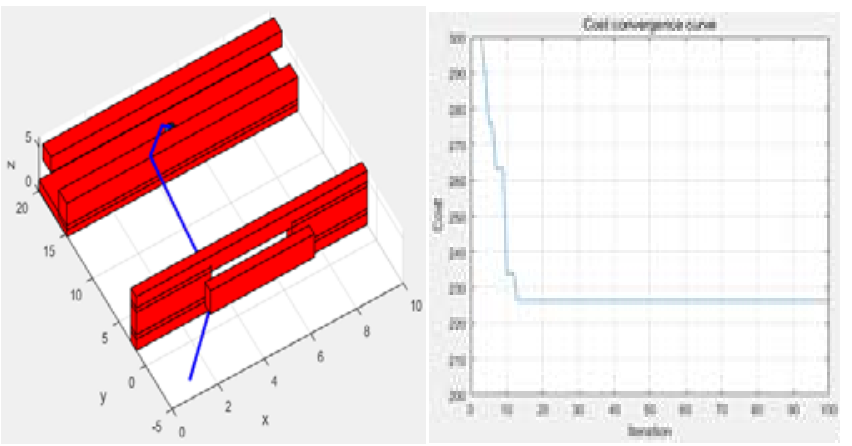

FIGURE III. PFACO PATH PLANNING AND ITS CONVERGENCE GRAPH

Compared figure 2 and figure 3 , the result shows that the PFACO has a better convergence than ACO thanks to the improved heuristic. What's more, the PFACO can find a better planning path with a shorter time, it can effective offer an obstacles-clear path to let UAV take mission and more reliable. 
TABLE I. THE PERFORMANCE COMPARISON BETWEEN PFACO AND ACO

\begin{tabular}{|l|c|c|c|}
\hline \multirow{2}{*}{$\begin{array}{c}\text { Table } \\
\text { Head }\end{array}$} & \multicolumn{3}{|c|}{ Cross Reference } \\
\cline { 2 - 4 } & Path Length & Cost & Duration \\
\hline ACO & 43.562 & 230.084 & 12.31 \\
\hline PFACO & 36.213 & 227.142 & 11.45 \\
\hline
\end{tabular}

Through the data in TABLE I, the result shows that PFACO has a better solution than ACO. The PFACO searching length is 36.213, duration is $11.45 \mathrm{~s}$. Compared with ACO, PFACO has more advantage in global searching with lower cost and time. What's more, PFACO complete inherit the ability of ACO, and can fit for any environment mission.

\section{CONCLUSIONS}

This paper main contributed on the foundation of the UAV automation navigation. Because of the compatibility and the disadvantage of ACO, we combine the ACO with APF to find a better path in shorter time. Through the connection in UAV with obstacles, the improved method uses the potential field as the information of heuristic. From the results, it shows that PFACO can effective overcome the local optimal and premature. What's more, speed up the searching ability. With the help of the evaporation strategy of MMAS improve the ability of global searching to have a better convergence. And the simulation also shows the safety and reliable path planning of PFACO. As to PFACO path planning is based in known environment, it can't handle the external environment. Therefore, the method needs further sensor to handle the random condition, and let automation navigation come true.

\section{ACKNOWLEDGEMENT}

In my researching time, it's my honor to work with the teachers and fellows whoever helped me. And I'll also give my thanks to the professor Xiaojia Zhou, Bin Yan. Last, I will give my thanks to doctor Ye for the advice and guidance during my researching time.

\section{REFERENCES}

[1]. Guo, Qing, Z. Zhang, and Y. Xu. "Path-planning of automated guided vehicle based on improved Dijkstra algorithm." Control and Decision Conference IEEE, 2017:7138-7143.

[2]. Raja, Rekha, and A. Dutta. "Path planning in dynamic environment for a rover using $A *$ and potential field method." International Conference on Advanced Robotics IEEE, 2017:578-582.

[3]. Kao, Chia Chia, C. M. Lin, and J. G. Juang. "Application of potential field method and optimal path planning to mobile robot control." IEEE International Conference on Automation Science and Engineering IEEE, 2015:1552-1554.

[4]. Fu, Si Yao, et al. "Path planning for unmanned aerial vehicle based on genetic algorithm." IEEE, International Conference on Cognitive Informatics \& Cognitive Computing IEEE, 2012:140-144

[5]. Chen, Zhiqiu, et al. "Modeling and Simulation of UAV Trajectory Planning on GAs." International Conference on Computer Modeling and Simulation IEEE Computer Society, 2009:7-9.

[6]. Lv, Zhanyong, and J. Cao. "Path planning methods of mobile robot based on new neural network." Control Conference IEEE, 2013:32223226.

[7]. Gan, Yi, et al. "Research on path planning for mobile robot based on ACO." Control and Decision Conference IEEE, 2017.

[8]. Ganganath, Nuwan, and C. T. Cheng. "A 2-Dimensional ACO-Based Path Planner for Off-Line Robot Path Planning." International
Conference on Cyber-Enabled Distributed Computing and Knowledge Discovery IEEE, 2013:302-307.

[9]. Ping, Gu, et al. "Adaptive ant colony optimization algorithm." International Conference on Mechatronics and Control IEEE, 2015:9598.

[10]. Shen, Yi, et al. "Study on Adaptive Planning Strategy Using Ant Colony Algorithm Based on Predictive Learning." 2009 Chinese Control and Decision Conference(2) 2009:3030-3035.

[11]. Zhang, Daqiao, et al. "UAV Path Planning Based on Chaos Ant Colony Algorithm." International Conference on Computer Science and Mechanical Automation IEEE, 2015:81-85.

[12]. Châari, Imen, et al. "smartPATH: A hybrid ACO-GA algorithm for robot path planning." IEEE Congress on Evolutionary Computation IEEE, 2012:1-8.

[13]. Shang, Ke, et al. "A GA-ACO hybrid algorithm for the multi-UAV mission planning problem." International Symposium on Communications and Information Technologies IEEE, 2014:243-248.

[14]. Shi, Chunxue, Y. Bu, and Z. Li. Path Planning for Deep Sea Mining Robot Based on ACO-PSO Hybrid Algorithm. IEEE, 2008.

[15]. Tan, Jianhao, et al. "Three-dimensional path planning based on ant colony algorithm with potential field For rotary-wing flying robot." IEEE International Conference on Information and Automation IEEE, 2015:2592-2597.

[16]. Chen, Ruey Maw, F. R. Hsieh, and D. S. Wu. "Heuristics based ant colony optimization for vehicle routing problem." Industrial Electronics and Applications IEEE, 2012:1039-1043.

[17]. Cekmez, U, M. Ozsiginan, and O. K. Sahingoz. "A UAV path planning with parallel ACO algorithm on CUDA platform." International Conference on Unmanned Aircraft Systems IEEE, 2014:347-354. 